\title{
Arabic Digits Recognition by Using Unified Binary Templates in The Digital Images
}

\author{
Sundus Khaleel Ebraheem \\ sunduskhaleel_2019@uomosul.edu.iq \\ College of Computer Sciences and Mathematics \\ University of Mosul, Mosul, Iraq
}

Received on: 18/02/2013

Accepted on: 16/09/2013

\begin{abstract}
The process of classification and recognition of the digits is very important and accurate issue, it should depend on a strong, adequate rule, because there are differences in the representation of the digits for various type of font, rather than to get homogeneous classification for group of digits to show the form or the constant shape for the digit from groups of font, where to be one shape represents each group of the shapes and sizes.

This paper proposed a new method for recognition images of Arabic digits with various types and size of font which captured by various kind of camera and various resolution depending on the characteristics of each digit which extracted from study of ten types of font with various size for each type. The characteristic of all shapes and sizes extracted for each digit. Then made a constant model for each digit to be compare with the digit image in any size or font type to recognize the digit in the image.

A database of ten templates (one for each digit) built, and these templates are basic and general to recognize the Arabic digits with various font and size. The new method applied for recognition of Arabic digits in the images of license plate of the passenger cars. the recognition percentage was $98 \%$.
\end{abstract}

Keywords: Image processing, Arabic Digits Recognition, Binary Templates, Arabic Digits detection, Digital Images, license plate recognition.

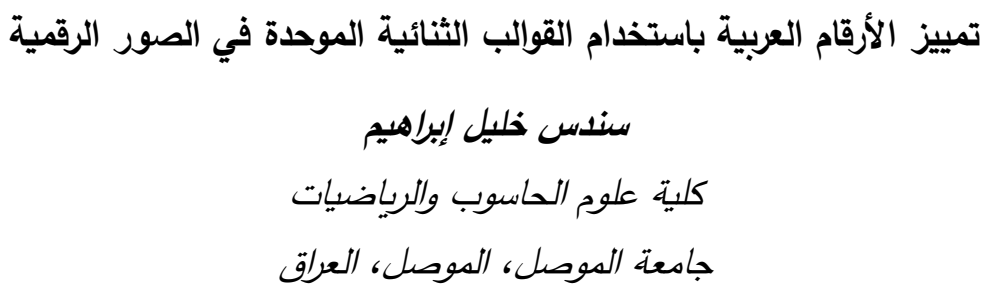

تاريخ قبول البحث: $2013 \mid 0916$

تاريخ استلام البحث: $2013 \backslash 02 \backslash 18$

$$
\begin{aligned}
& \text { الملخص } \\
& \text { إن عملية تصنيف وتمييز الأرقام من الأمور المهمة والدقيقة ويجب أن تعتمد على قاعدة رصينة وصحيحة } \\
& \text { لوجود الاختلاف في تمثيل الأرقام في نوع الخط المستخدم، ومن اجل الحصول على تصنيفات متجانسة لمجموعة } \\
& \text { الأرقام لإظهار الهيأة النظامية أو الثكل الثابت للرقم من مجاميع الخطوط بحيث يصبح هنالك شكل واحد يمثل كل } \\
& \text { مجموعة من أشكال وأحجام كل رقم. }
\end{aligned}
$$


تم في هذا البحث اقتراح طريقة جديدة لتمييز صور الأرقام العربية بأنواع وأحجام مختلفة من الخط ملتقطة بأنواع مختلفة من الكاميرات وبدقات مختلفة بالاعتماد على صفات شكل كل رقم التي تم استخلاصها من دراسة 10 أنواع من الخطوط وبأحجام مختلفة لكل نوع. حيث تم استخلاص صفات تجمع بها كل أشكال الرقم باختلاف

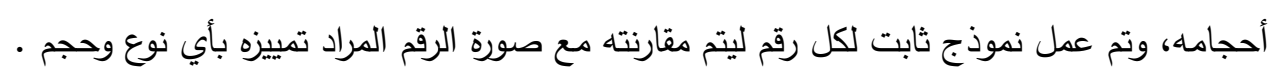
تم بناء قاعدة بيانات تتكون من 10 قوالب ( قالب واحد لكل رقم)وتكون هذه القوالب أساسية وعامة لتمييز الأرقام العربية بمختلف أثكالها وأحجامها. وتم تطبيق الطريقة الجديدة في تمييز الأرقام العربية في صورة لوناء لوحات

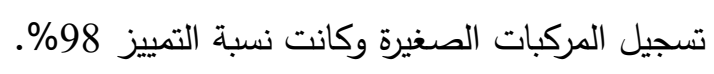
الكلمات المفتاحية: معالجة الصور، تمييز الأرقام العربية، القوالب الثنائية، اكتثاف الأرقام العربية، الصور الرقمية، تمييز لوحة الترخيص.

1

إن مسائل تمييز الصور تكون عادة صعبة الحل باستخدام البيانات غير المعالجة. لتحسين عملية التمييز

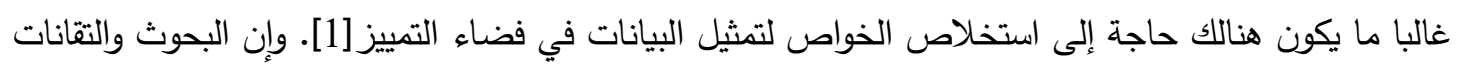
الخاصة بعلم تمييز الأنماط تهدف إلى إيجاد أو التعرف على أنماط أو هياكل محددة في الصور الرقمية (إثارة

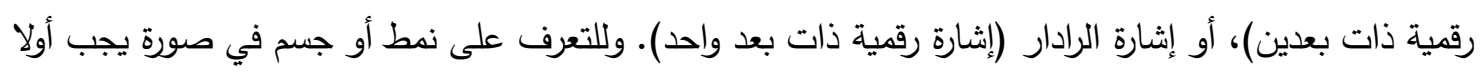

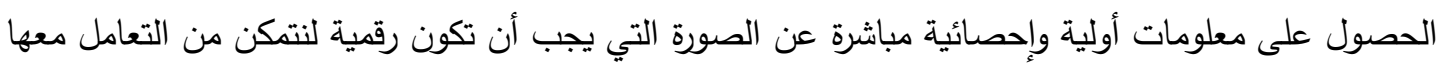
بالحاسوب. كذلك يجب تصنيف المعلومات الموجودة في الصورة لتسهيل عمليات لاحقة على الصورة مثل استخلاص المعلومات[2].

إن مراحل التعرف على أي نمط تمر بمرحلتين: الأولى مرحلة التعلم Learning، والثانية مرحلة التصنيف

أو التمييز .Classification or recognition يوجد طرائق أساسية مستخدمة في تمييز الأنماط منها] [2]: طريقة قالب المطابقة [3] والطريقة الإحصائية وطريقة تمييز الأنماط باستخدام الثبكات العصبية[4] وطريقة تمييز الأنماط باستخدام العزوم [5]...الخ

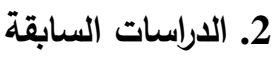

هنالك العديد من الطرائق لتقطيع الصور إلى مناطق والتي يتم تحليلها بالاعتماد على الثكل او الحجم أو الو

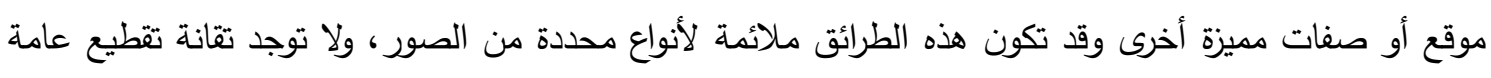

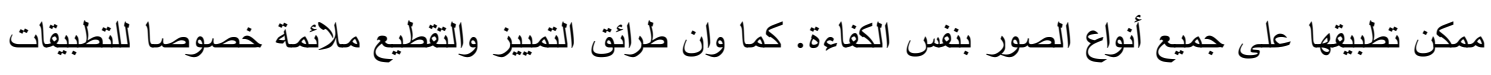

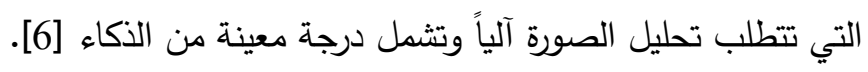
وقد كان هذا الحقل موضع دراسة من قبل الكثير من الباحثين، وتوجد عدة طرائق لاستخلاص صفاء

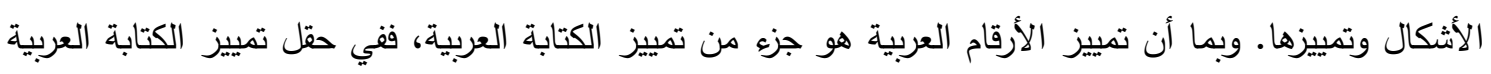

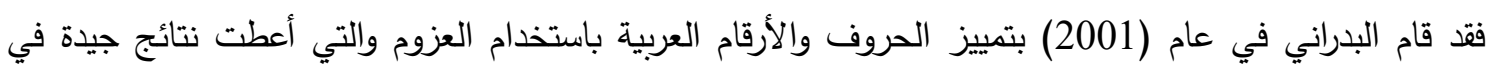
التمييز[7] كما ذكر الباحث. وفي عام(2002) ميز الباحث إبراهيم الحروف العربية المطبوعة والمكتوبة بخط اليد باستخدام الشبكات العصبية[8]. اما في عام (2004) فقد قام الباحث ذنون بتمييز الحروف المطبوعة باستخدام

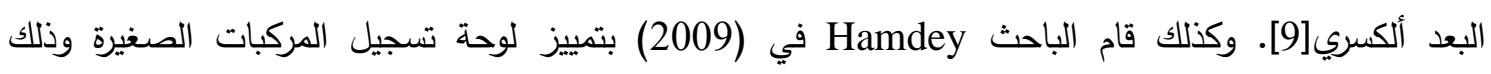
بالاعتماد على صفات شكل الرقم [10]. وفي العام ذاته قام الباحث الاهدل بتطبيق شبكة الانتشار العكسي لتمييز الأرقام العربية المكتوبة يدويا[11]. وكذلك في العام نفسه قام كل من الزبيدي والدليمي باستخدام شبكة 
النيوكوكنترون نظاماً صورياً لتمييز الصور الداخلة التي تمثل صور الأرقام العربية المكتوبة يدويا من خلال محاكاة الخلية البايولوجية العاملة في عين الإنسان[12]. وفي عام (2010) قدم Gohil طريقة لقطع لوحة تسجيل المركبة بالاعتماد على المدرج التكراري[13]. وقدم الباحث طليع وآخرون عام (2011) طريقة جديدة لتمييز الحروف العربية باختلاف أحجامها والمتحركة منها (التي تحتوي على الحركات مثل الفتحة والضمة و ...الخ) وتم استخلاص ولاص صفات الحروف باستخدام مصفوفة حدوث المشاركة لكل صورة والذي يمكن تطبيقه أيضا على الأرقام العربية والانكليزية وتمت عملية التمييز باستخدام شبكة الانتشار العكسي(back propagation) وشبكة المدرك (4erceptron) [4) كما قام كل من الطائي وعتاب في العام نفسه ببناء منظومة تمييز آلية لتمييز الأرقام العربية

المكتوبة يدويا باستخدام الدالة المميزة التربيعية والمعتمدة على الأسلوب الإحصائي في عملية التمييز [14].

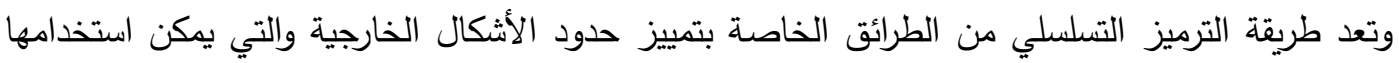
في تمييز الأرقام أيضا. ففي عام (2012) تمت دراسة مشاكل هذه الطريقة كإحدى طرائق التمييز واقترح كل من العبيدي والعطيوي طريقة لحل مشكلة تغيير الحجم[15]. إن مشاكل تمييز الأرقام العربية باستخدام بعض الطرائق السابقة يمكن أن تلخص كما يأتي: 1- إن الطرائق السابقة كانت تعمل على نوع معين من الخط ولا تعمل على نوع آخر .

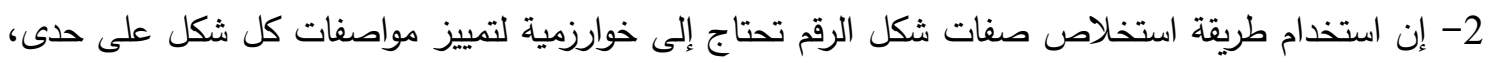
حيث تحتاج الخوارزمية إلى تحديد نقطة بداية تتبع الثكل وتحديد اتجاه الحركة بالنسبة لتمييز الرقم 2 عن الرقم 6 وكذلك التمييز بين الرقم 7 والرقم 8. وكذلك تحتاج إلى إيجاد اتجاه الزوايا للتمييز بين الرقم 3 و4 فضلاه عن التهن

$$
\text { تحديد الفراغات المستديرة للتمييز بين الرقم } 5 \text { والرقم } 9 .
$$
3- من خلال التجارب التي تم القيام بها لتمييز صور الأرقام باستخدام الثبكات العصبية (شبكة الانتشار العكسي

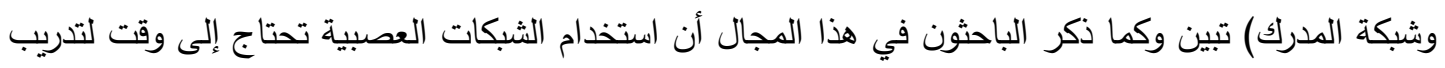

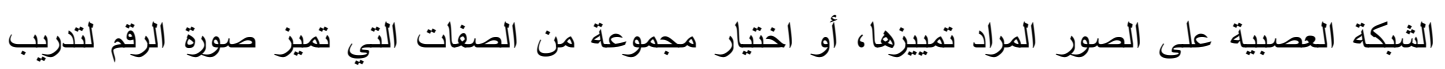

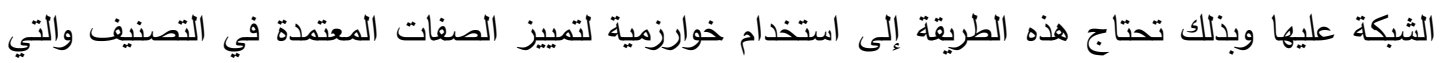
سيتم إدخالها فيما بعد إلى الثبكة العصبية الاصطناعية للتدريب، فضلا عن أن الثبكات العصبية تميز

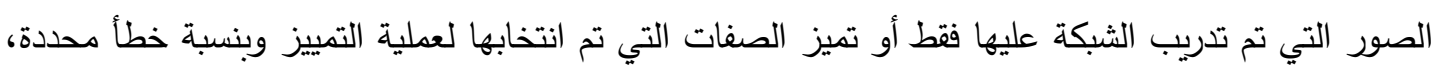
أي أن استخدام الثبكات العصبية لتمييز صور جديدة تم التقاطها بكاميرا أخرى أو بنفس الكاميرا مع تغيير

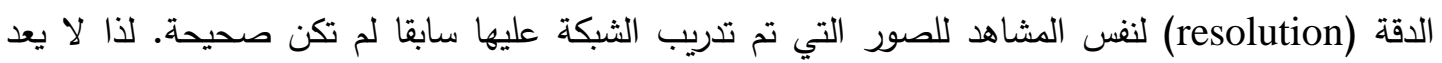
استخدام الثبكات العصبية في تمييز الأرقام بأنواع مختلفة من الخط أو تمييز صور الأرقام المتتطة بكاميرات

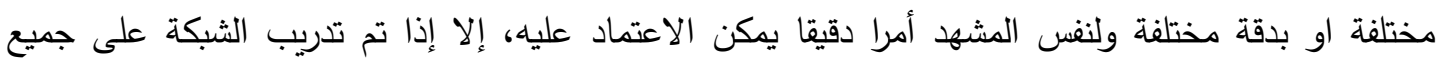
الاحتمالات بكافة الأحجام واللقطات. 4- اثبت الباحثون أن استخدام العزوم الثابتة في تمييز الصور الرقمية يكون مقاوم للتشوهات والتغير بالحجم

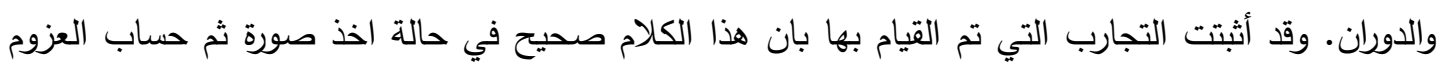
لها ثم تدويرها او تغيير حجمها أو إضافة التشوهات لها باستخدام الحاسوب ثم حساب العزوم مرة أخرى. ولكن

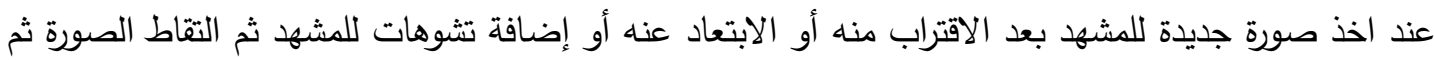

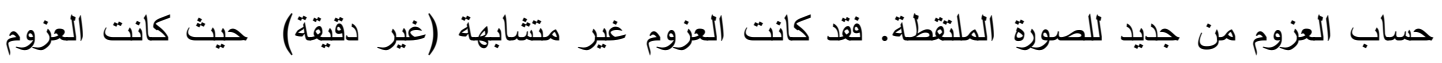
حساسة لهذه التغيرات، لذا كان من غير الممكن اعتمادها في عملية تمييز الأرقام بكل إحجامها وأثكالها. 
ولازالت البحوث مستمرة في هذا الموضوع وخاصة تمييز الأرقام العربية لاستخدامها مثلا في تمييز لوحة

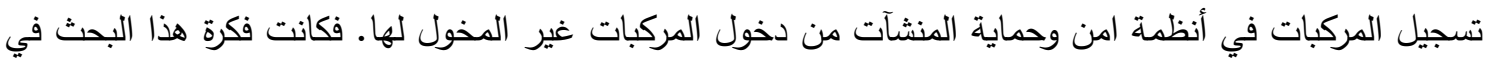
اقتراح طريقة تمييز جديدة تثمل كل أنواع وأحجام كتابة الأرقام العربية.

\section{3. - الهـف من البحث}

إن الهدف من البحث هو إيجاد طريقة تكون مناسبة لتمييز الأرقام العربية في الصور الرقمية بكافة أحجامها وأثكالها، وذلك بدراسة أشكال الأرقام العربية وبأحجام مختلفة وإبراز أهم نقاط الالتقاء بين هذه الأشكال لكل رقد وذلك بتحويلها إلى مصفوفة بيانات تعتمد تعريف الصفوف والأعمدة للوصول إلى صيغة تضم خواص (features) أو صفات مميزة (attributes) تصبح قاعدة أساسية عامة تسهم مساهمة فعالة لتكون إحدى طرائق تمييز الأرقام. وهذه الطريقة تكون قادرة على التمييز بين الصور الرقمية الخاصة بالأرقام العربية حتى لو ظهرت تلك الصور بظروف صورية مختلفة عن الصور أو النماذج المرجعية.

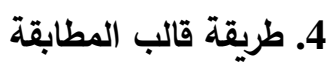

إن مرحلة التعليم في هذه الطريقة تقوم على تخزين مجموعة من القوالب Templates أو النماذج Prototypes (قالب من كل صنف) في الحاسوب كما موضح في الثكل(1)(4,2].

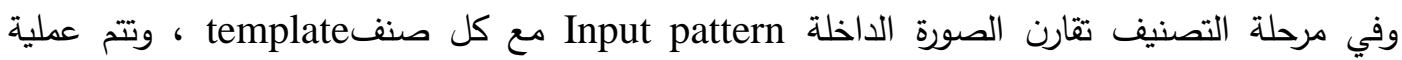
pixel by pixel المطابقة بخزن الصورة الداخلة على شكل مصفوفة وتقارن مع القوالب الموجودة في الجهاز وتعطي قيمة للمقارنة.

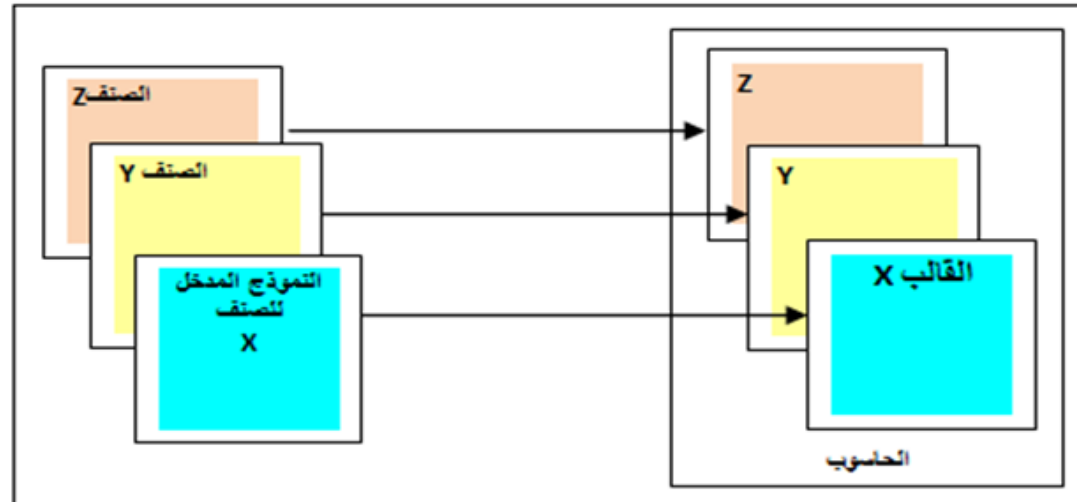

الثُكل(1) (1): مجموعة من القولب

تعد هذه الطريقة طريقة سهلة، والصعوبة الوحيدة في هذه الطريقة هي الاختيار الجيد للقوالب من كل

[2] صنف بالإضافة إلى تحديد معايير المقارنة وخصوصاً لو كانت الصورة الداخلة تحمل تثوهات 5. الآلية المستخدمة في التطبيق إن آلية العمل المستخدمة في هذا البحث تتمثل بعدة خطوات أساسية وهي كما يأتي:

1.5

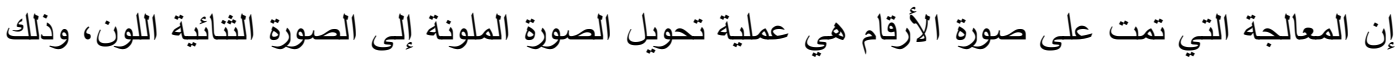

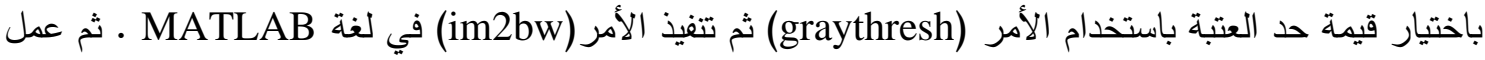


تمديد(dilate) مرة واحدة للصورة وذلك لتحسين الصورة بملء الفراغات في الصورة وتحديد حافات الرقم بربط

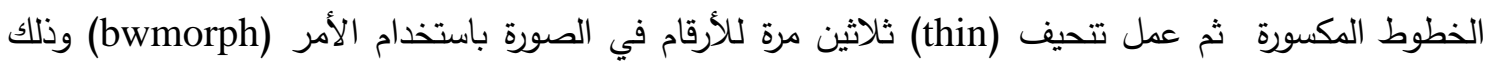
لإزالة المعلومات الزائدة والحصول على المعلومات المهمة الخاصة بثكل كل رقم، انظر المقطع البرمجي الاتي: $\mathrm{a}=$ imread(filename); level $=$ graythresh $(\mathrm{a})$; $\mathrm{BW}=\mathrm{im} 2 \mathrm{bw}(\mathrm{a}, \mathrm{level})$; figure;imshow(BW)

$\mathrm{BW}=$ imcomplement $(\mathrm{BW}) ; \%$ to inverse color

BW=bwmorph(BW,'dilate',1);

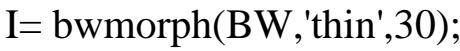

2.5

إن الآلية المستخدمة في عملية القطع التي ستوضح لاحقا بالتفصيل في فقرة " الخوارزمية المقترحة في

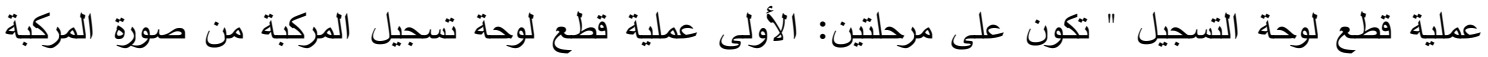
الأصلية بالاعتماد على الإطار المحيط بلوحة التسجيل ثم فصل جزء الرقم عن الجزء الخاص باسم البلد والمحافظة باستخدام الددرج التكراري. والمرحلة الثانية عملية فصل كل رقم من الصورة بالاعتماد على الفراغات، حيث تم فصل الأعمدة التي لا تحتوي نقاط مضيئة عن بعضها باعتبارها الفاصل بين الأرقام في الصورة (أي نهاية صورة

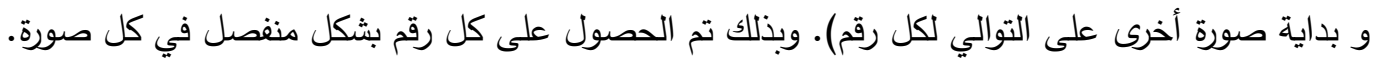
3.5

إن لكل كائن في الصورة صفات معينة يمكن اكتثافه من خلالها وذلك بتحديد حواف الكائن ثم تتبع حدوده الخارجية ثم تمييز هذه الصفات التي تخص كل كائن بشكل مستقل عن الأخر •

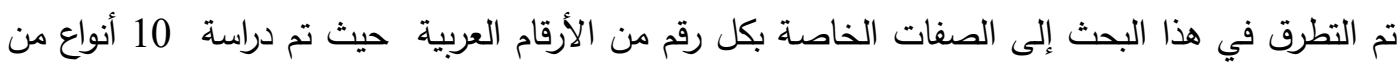
خطوط الأرقام وبأحجام مختلفة (72،36،28) اعتيادية وغامقة وتبين أن هذه الأرقام بمختلف أشكالها وأحجامها

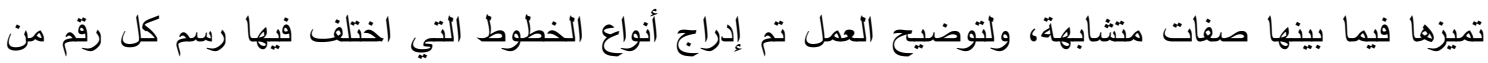
الأرقام. انظر الجدول(1) الذي يوضح 3 أنواع من خطوط الأرقام وبحجم 36 التي لوحظ فيها اختلاف رسم كل رقم من الأرقام بشكل واضح فضلا عن خط اليد، والجدول (2) يين بشكل واضح اختلاف أشكال الأرقام بعد عملية 


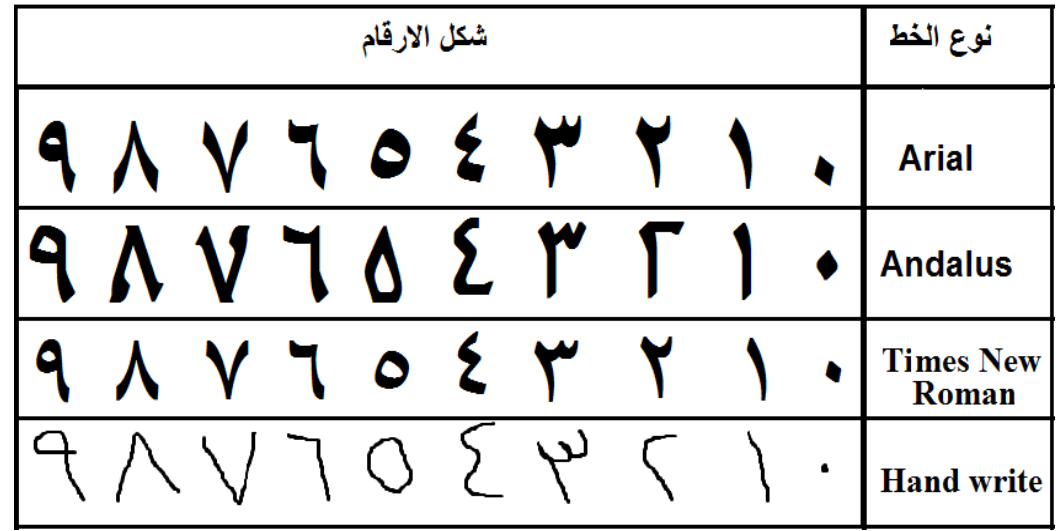

الجدول (2): صور الأرقام بعد عملية التنحيف.

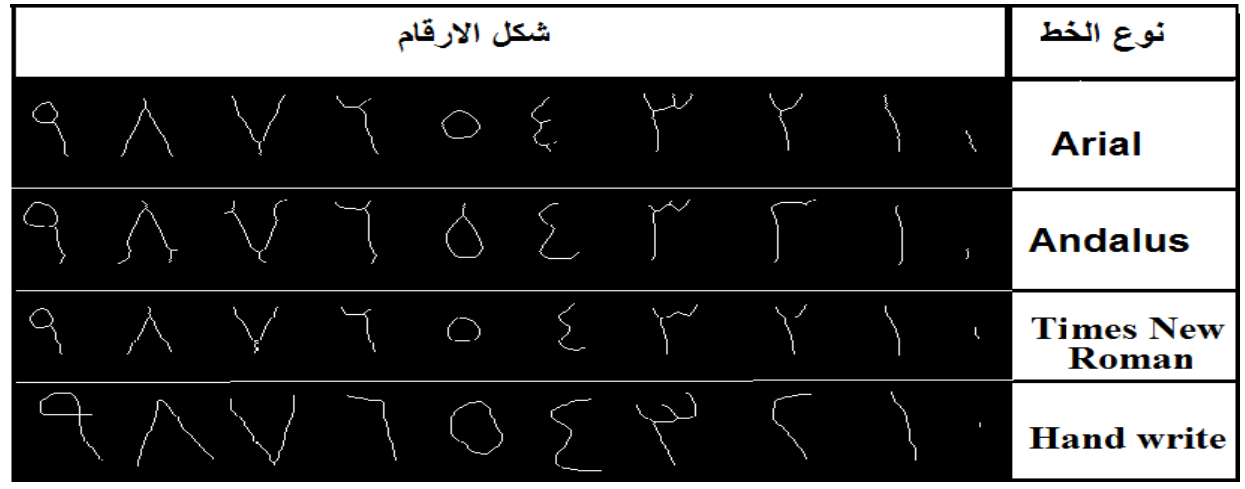

تم عمل بيئة ثابتة لكل الأرقام في اللوحة الواحدة تمثلت هذه البيئة كما يأتي:

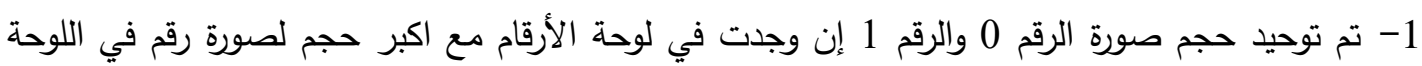
الواحدة وذلك لخلق بيئة واحدة لكل الأرقام يمكن أن تعتمد في عملية استخلاص صفات التفات الثكل بعد قطعها

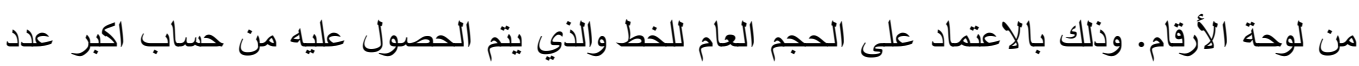

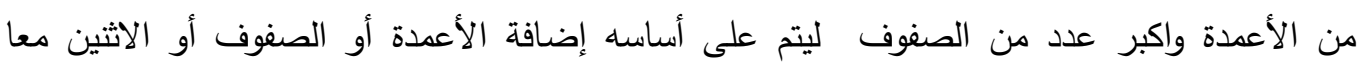
للصورة الأقل عدد من الأعمدة والصفوف، للحصول على الحجم الصحيح لهذه الصورة.

2- تقسيم الصورة إلى ستة عشر جزء (4×4)، انظر الجدول (3).

الجدول (3): يبين تقسيم كل صورة إلى 16 جزء.

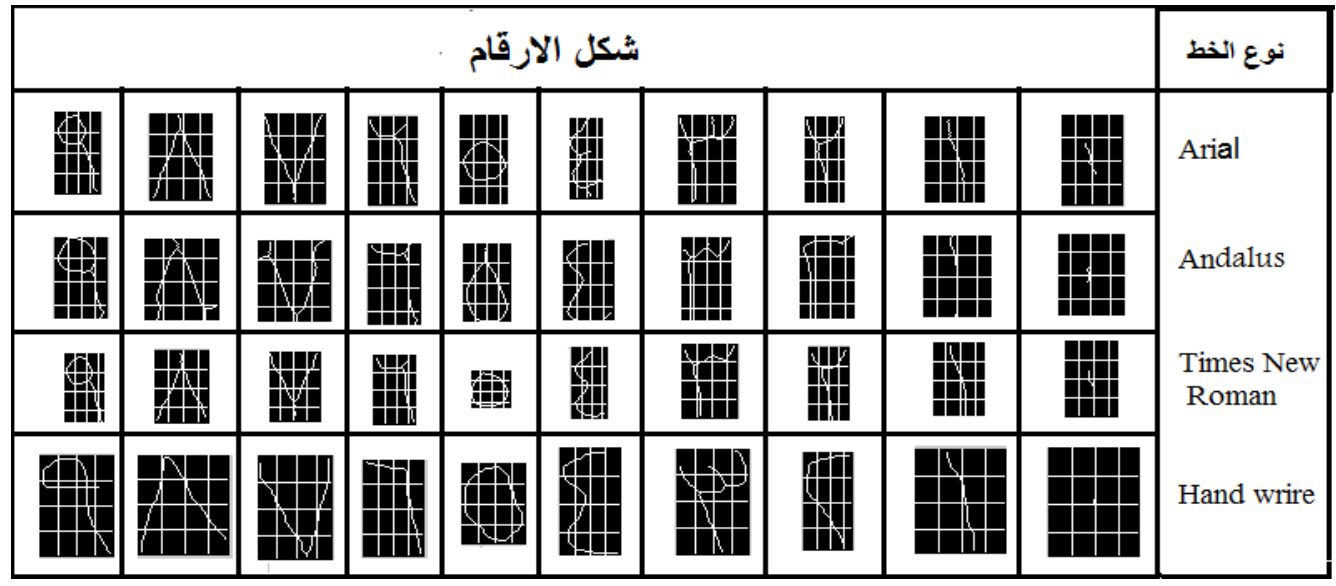


3- تحويل الصورة إلى صيغة الأرقام الثنائية بطول 16 عنصر أي 4×4. ويتم ذلك بتحويل كل جزء من الأجزاء الستة عشر إلى الرمز '1' إذا احتوى الجزء الواحد على نقطة ضوئية واحدة أو أكثر ، أما إذا لم لم

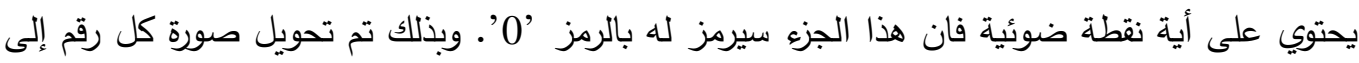

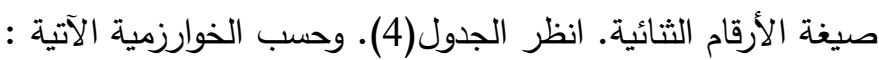
الجدول (4): يبين الصيغة الثنائية لصور الأرقام.

\begin{tabular}{|c|c|c|c|c|c|c|c|c|c|c|}
\hline 9 & $\Lambda$ & $V$ & 9 & $\theta$ & $\xi$ & $\boldsymbol{p}$ & 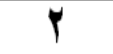 & 1 & " & نوع الخط \\
\hline $\begin{array}{llll} & 1 & 1 & 0 \\
1 & 1 & 1 & 0 \\
0 & 0 & 1 & 0 \\
0 & 0 & 0 & 1\end{array}$ & $\begin{array}{llll}0 & 1 & 0 & 0 \\
0 & 1 & 1 & 0 \\
1 & 1 & 1 & 1 \\
1 & 0 & 0 & 1\end{array}$ & $\begin{array}{llll}1 & 0 & 0 & 1 \\
1 & 1 & 1 & 1 \\
0 & 1 & 1 & 1 \\
0 & 1 & 1 & 0 \\
0 & 1 & 0 & 0\end{array}$ & $\begin{array}{llll} & 1 & 1 & 0 \\
0 & 0 & 1 & 0 \\
0 & 0 & 1 & 1 \\
0 & 0 & 0 & 1\end{array}$ & $\begin{array}{llll}0 & 0 & 0 & 0 \\
1 & 1 & 1 & 1 \\
1 & 1 & 1 & 1 \\
0 & 0 & 0 & 0\end{array}$ & $\begin{array}{llll}1 & 1 & 1 & 0 \\
1 & 1 & 1 & 0 \\
1 & 1 & 0 & 0 \\
1 & 1 & 1 & 0 \\
1 & 1 & 1\end{array}$ & $\begin{array}{llll}1 & 1 & 1 & 1 \\
1 & 1 & 0 & 0 \\
0 & 1 & 0 & 0 \\
0 & 1 & 0 & 0\end{array}$ & $\begin{array}{lllll}1 & 0 & 1 & 1 \\
0 & 1 & 1 & 0 \\
0 & 0 & 1 & 0 \\
0 & 0 & 1 & 0 \\
0 & 0 & 0\end{array}$ & $\begin{array}{llll}0 & 1 & 0 & 0 \\
0 & 1 & 1 & 0 \\
0 & 0 & 1 & 0 \\
0 & 0 & 1 & 0\end{array}$ & $\begin{array}{llll}0 & 0 & 0 & 0 \\
0 & 1 & 0 & 0 \\
0 & 1 & 1 & 0 \\
0 & 0 & 0 & 0\end{array}$ & \multirow{2}{*}{$\begin{array}{l}\text { Arial } \\
\text { Andalus }\end{array}$} \\
\hline $\begin{array}{llll}1 & 1 & 1 & 1 \\
1 & 1 & 1 & 1 \\
0 & 0 & 0 & 1 \\
0 & 0 & 0 & 1\end{array}$ & $\begin{array}{llll}0 & 1 & 1 & 0 \\
0 & 1 & 1 & 0 \\
1 & 0 & 1 & 0 \\
1 & 0 & 0 & 1\end{array}$ & $\begin{array}{llll}1 & 0 & 0 & 1 \\
1 & 1 & 0 & 1 \\
0 & 1 & 1 & 1 \\
0 & 1 & 1 & 1\end{array}$ & $\begin{array}{llll}1 & 1 & 1 & 1 \\
0 & 0 & 0 & 1 \\
0 & 0 & 0 & 1 \\
0 & 0 & 0 & 1\end{array}$ & $\begin{array}{llll}0 & 0 & 1 & 0 \\
1 & 1 & 1 & 0 \\
1 & 0 & 0 & 1 \\
1 & 1 & 1 & 1\end{array}$ & $\begin{array}{llll}1 & 1 & 0 & 0 \\
1 & 1 & 0 & 0 \\
1 & 1 & 0 & 0 \\
1 & 1 & 1 & 1\end{array}$ & $\begin{array}{llll}1 & 1 & 1 & 1 \\
1 & 0 & 0 & 0 \\
1 & 0 & 0 & 0 \\
1 & 0 & 0 & 0\end{array}$ & $\begin{array}{llll}1 & 1 & 1 & 1 \\
1 & 0 & 0 & 0 \\
1 & 0 & 0 & 0 \\
1 & 0 & 0 & 0\end{array}$ & $\begin{array}{lllll}0 & 1 & 0 & 0 \\
0 & 1 & 1 & 1 \\
0 & 0 & 1 & 0 \\
0 & 1 & 1 & 0\end{array}$ & $\begin{array}{llll}0 & 0 & 0 & 0 \\
0 & 1 & 0 & 0 \\
0 & 1 & 0 & 0 \\
0 & 0 & 0 & 0\end{array}$ & \\
\hline $\begin{array}{llll}1 & 1 & 1 & 0 \\
1 & 1 & 1 & 0 \\
0 & 1 & 1 & 1 \\
0 & 0 & 1 & 1\end{array}$ & $\begin{array}{llll}0 & 1 & 0 & 0 \\
0 & 1 & 1 & 0 \\
1 & 1 & 1 & 1 \\
1 & 0 & 0 & 1\end{array}$ & $\begin{array}{llll}1 & 0 & 0 & 1 \\
1 & 1 & 1 & 1 \\
0 & 1 & 1 & 0 \\
0 & 0 & 1 & 0\end{array}$ & $\begin{array}{llll}1 & 1 & 1 & 1 \\
0 & 0 & 1 & 0 \\
0 & 0 & 1 & 1 \\
0 & 0 & 0 & 1\end{array}$ & $\begin{array}{llll}0 & 1 & 1 & 0 \\
1 & 0 & 0 & 1 \\
1 & 0 & 0 & 1 \\
1 & 1 & 1 & 0\end{array}$ & $\begin{array}{llll}1 & 1 & 0 & 0 \\
1 & 1 & 0 & 0 \\
1 & 1 & 0 & 0 \\
1 & 1 & 1 & 1\end{array}$ & $\begin{array}{llll}1 & 1 & 1 & 1 \\
1 & 1 & 0 & 0 \\
0 & 1 & 0 & 0 \\
0 & 1 & 0 & 0\end{array}$ & $\begin{array}{llll}1 & 0 & 0 & 1 \\
0 & 1 & 1 & 0 \\
0 & 1 & 1 & 0 \\
0 & 0 & 1 & 0\end{array}$ & $\begin{array}{lllll}0 & 1 & 0 & 0 \\
0 & 1 & 1 & 0 \\
0 & 0 & 1 & 0 \\
0 & 0 & 1 & 0\end{array}$ & $\begin{array}{lllll}0 & 0 & 0 & 0 \\
0 & 1 & 0 & 0 \\
0 & 1 & 0 & 0 \\
0 & 0 & 0 & 0\end{array}$ & \multirow[t]{2}{*}{$\begin{array}{l}\text { Times New } \\
\text { Roman }\end{array}$} \\
\hline $\begin{array}{llll}1 & 1 & 1 & 0 \\
1 & 1 & 1 & 1 \\
0 & 0 & 1 & 1 \\
0 & 0 & 0 & 1\end{array}$ & $\begin{array}{llll}0 & 1 & 0 & 0 \\
1 & 1 & 1 & 0 \\
1 & 0 & 1 & 1 \\
1 & 0 & 0 & 1\end{array}$ & $\begin{array}{llll}1 & 0 & 0 & 1 \\
1 & 1 & 0 & 1 \\
0 & 1 & 0 & 1 \\
0 & 0 & 1 & 0\end{array}$ & $\begin{array}{llll}1 & 1 & 1 & 0 \\
0 & 0 & 0 & 1 \\
0 & 0 & 0 & 1 \\
0 & 0 & 0 & 1\end{array}$ & $\begin{array}{llll}1 & 1 & 1 & 0 \\
1 & 0 & 1 & 1 \\
1 & 0 & 0 & 1 \\
0 & 1 & 1 & 1\end{array}$ & $\begin{array}{llll}1 & 1 & 1 & 0 \\
1 & 1 & 0 & 0 \\
1 & 1 & 0 & 0 \\
1 & 1 & 1 & 1\end{array}$ & $\begin{array}{llll}1 & 1 & 1 & 1 \\
1 & 1 & 1 & 1 \\
0 & 1 & 0 & 0 \\
0 & 0 & 1 & 0\end{array}$ & $\begin{array}{llll}1 & 1 & 1 & 1 \\
1 & 0 & 0 & 0 \\
0 & 1 & 1 & 0 \\
0 & 0 & 1 & 0\end{array}$ & $\begin{array}{lllll}0 & 1 & 0 & 0 \\
0 & 1 & 1 & 0 \\
0 & 0 & 1 & 0 \\
0 & 0 & 1 & 0\end{array}$ & $\begin{array}{lllll}0 & 0 & 0 & 0 \\
0 & 0 & 0 & 0 \\
0 & 0 & 0 & 0 \\
0 & 0 & 0 & 0\end{array}$ & \\
\hline
\end{tabular}

أ- تجزئة الصورة الثنائية لكل رقم إلى 16 جزء وذلك بتحديد أرقام السطور و أرقام الأعمدة التي سيتم قطعها

$$
\begin{aligned}
& \text { لكل جزء من صورة الرقم. وحسب الإيعاز الأتي: } \\
& \text { Part = image(r1:r2 , c1:c2); }
\end{aligned}
$$

حيث تمثل image صورة الرقم المراد تجزئته وr1 رقم السطر الذي يمثل بداية مقطع الصورة وr2 رقم السطر الذي يمثل نهاية مقطع الصورة وc1 هو رقم العامود الذي يمثل بداية مقطع الصورة وc2 رقم العامود الذي لهي يمثل نهاية مقطع الصورة. ب- استخدام الأمر (sum) لإيجاد مجموع عناصر كل جزء من الأجزاء الستة عشر، كما في الإيعاز الأتي: $\mathrm{S}=\operatorname{sum}(\operatorname{sum}($ part $))$; ج- توضع نتيجة جمع كل جزء من الصورة في الحقل المقابل لله في مصفوفة ثنائية تم تكوينها بحجم (4×4). حيث توضع قيمة ’1' في الحقل إذا كانت نتيجة الجمع اكبر من صفر، وإلا ستكون قيمة

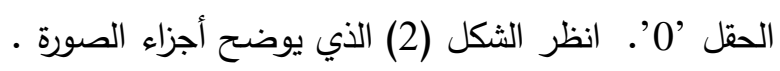
الشكل(2) الأجزاء الستة عشر للصورة

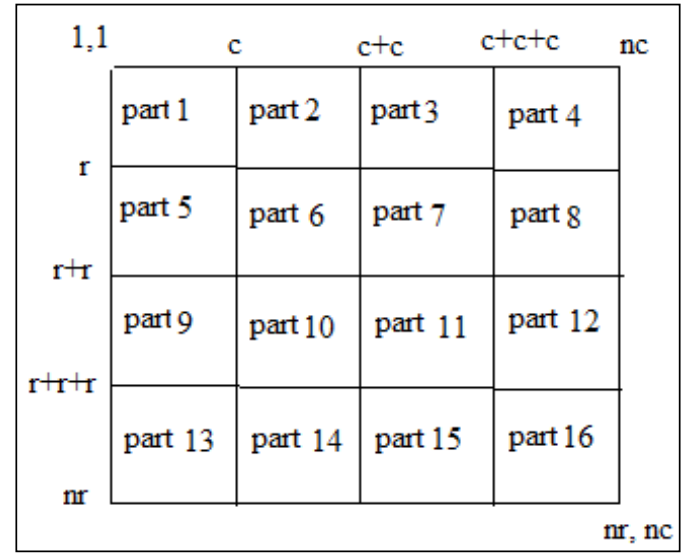


6. القوالب الثنائية الموحدة المقترحة

بما أن عملية تصنيف وتمييز الأرقام يجب أن تعتمد على قاعدة لابد أن تكون رصينة وصحيحة لوجود الاختلاف في تمثيل الأرقام، ومن اجل الحصول على تصنيفات متجانسة لمجموعة من العناصر لإظهار الهيأة النظامية أو الثكل الثابت للمجاميع النهائية بحيث يصبح هنالك شكل واحد يمثل كل مجموعة من أثكال وأحجام كل رقم ومن ملاحظة الجدول (4) يمكن استخلاص صفات موحدة لكل رقم والمبينة في الجدول (5) التي تمثل قاعدة البيانات الأساسية العامة التي سيتم المقارنة معها لتمييز الأرقام بكل أشكالها وأحجامها. الجدول (5): قوالب الأرقام العربية المستخدمة في قاعدة البيانات(الرمز "-" يشير إلى أن القيمة مهملة).

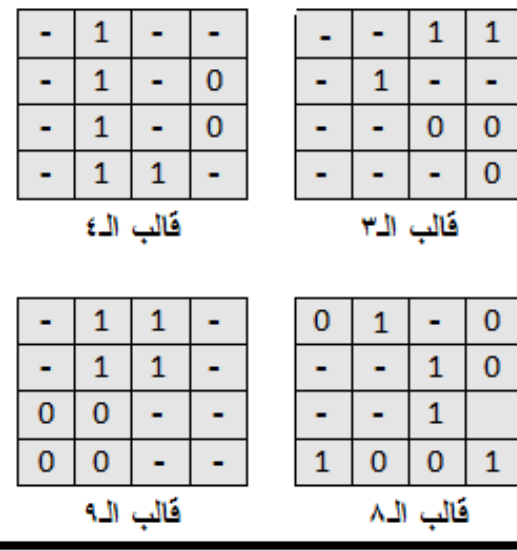

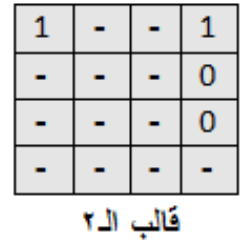

\begin{tabular}{|l|l|l|l|}
\hline 1 & 0 & - & 1 \\
\hline 1 & - & - & - \\
\hline- & - & - & 1 \\
\hline 0 & - & - & 0 \\
\hline \multicolumn{5}{|c|}{ Vـا } \\
\hline
\end{tabular}
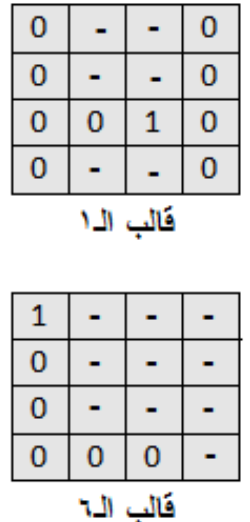

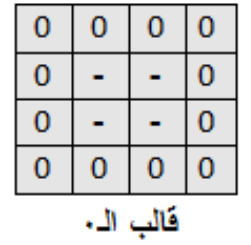

\begin{tabular}{|c|c|c|c|}
\hline- & - & - & - \\
\hline 1 & - & - & - \\
\hline 1 & - & - & 1 \\
\hline- & - & - & - \\
\hline \multicolumn{5}{|c}{} \\
\hline
\end{tabular}

7. تطبيق الخوارزمية المقترحة

إن الخوارزمية المقترحة تتقسم إلى جزأين:

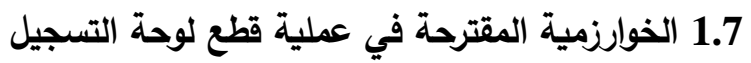
تم تطبيق الخوارزمية المقترحة على صور لوحة تسجيل المركبات، لذا كان هنالك حاجة لعمل برنامج لقطع

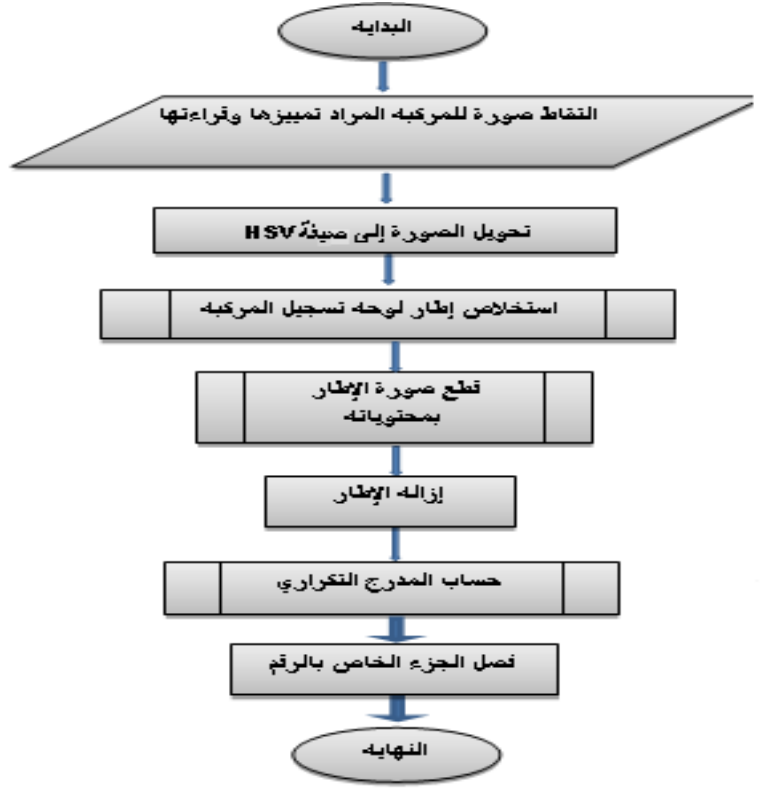

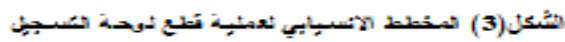

صورة لوحة تسجيل المركبة عن صورة المركبة. إن لوحة تسجيل المركبة التي سيتم تمييزها

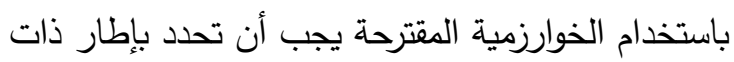
لون معين وليكن اخضر مثلا، حيث تم تمييز موقع لإن الإطار الأخضر باستخدام حد العتبة، وذلك بتحويل

Hue, Saturation, ) HSV الصورة إلى صيغة Value وكانت قيمة حد العتبة بين القيمتين (-0.50) 0.20) في المستوي_Hue والقيمة بين (0.45-0.90) في المستوي Saturation والقيمة بين (0.40-1.00) في المستوي Value، هذه القيم تعطي تدرجات اللون الأخضر كلها تقرببا مع اختلاف نسبة الإضاءة في

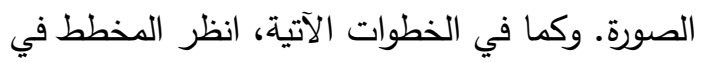

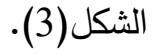


1- التقاط صورة المركبة المراد تمييزها.

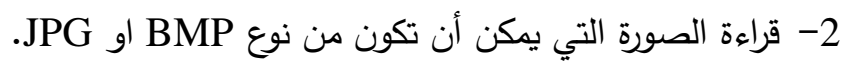

3- تحويل الصورة إلى نظام

4- استخدام حدود العتبة لاستخلاص الإطار الأخضر .

5- قطع صورة الإطار بمحتوياته.

6- إزالة الإطار من الصورة للحصول على صورة رقم المركبة فقط.

7- حساب ورسم المدرج التكراري (histogram) لتمثيل النقاط المضيئة في كل عامود.

8- فصل جزء الأرقام عن جزء البلد والمحافظة، وذلك بحساب اكبر مسافة بين القيم في المدرج التكراري تقسم

اللوحة إلى جزأين. حيث يعد الجزء إلى اليمين هو رقم المركبة والجزء إلى اليسار هو اسم البلد

والمحافظة. وبذلك تم الحصول على رقم لوحة المركبة بثكل منفصل عن اسم البلد والمحافظة.

9

2.7 الخوارزمية المقترحة في تمييز الأرقام

فيما يأتي خطوات الخوارزمية المقترحة لتمييز الأرقام العربية والموضحة في المخطط الانسيابي في

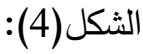

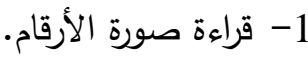

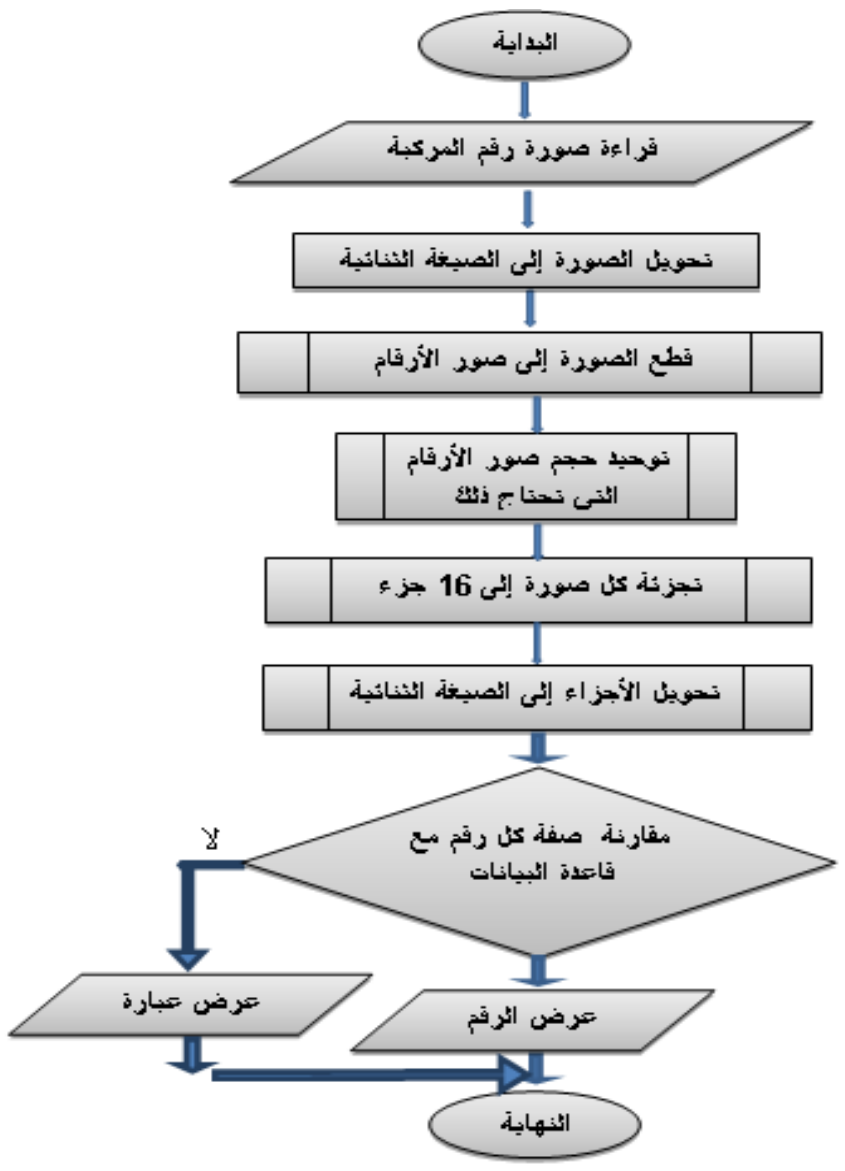

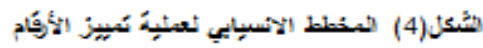

$$
\begin{aligned}
& \text { 2- تحويل الصورة الملونة إلى الصورة } \\
& \text { الثنائية. }
\end{aligned}
$$

7- تقسيم كل صورة ناتجة من الخطوة السابقة

$$
\text { إلى } 16 \text { جزء (4) (4). }
$$

8- تحويل أقسام الصور إلى صيغة الأرقام

الثنائية لتثبيت صفات صورة الرقم.

9- مقارنة كل صيغة ثنائية ناتجة من الخطوة

السابقة مع القوالب في قاعدة البيانات

العامة لإيجاد الرقم الصحيح.

10- طباعة الرقم أو عرض عبارة خطأ في حالة

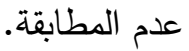

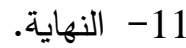


8.

تم تطبيق الخوارزمية المقترحة على صور لوحة تسجيل المركبات الصغيرة وكما في المثال الأتي:

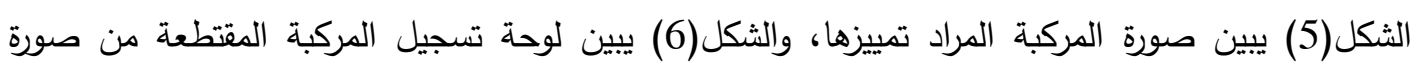
المركبة بالاعتماد على الإطار الأخضر ، الثكل(7) يوضح الصورة بعد تحويلها إلى الصورة الثنائية، ثم يتم

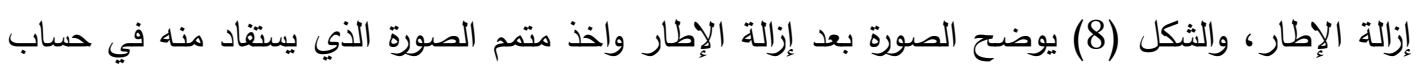

المدرج التكراري المبين في الثكل(9) حيث تكون النقاط المضيئة مساوية لـ1 والخلفية تساوي صفر .

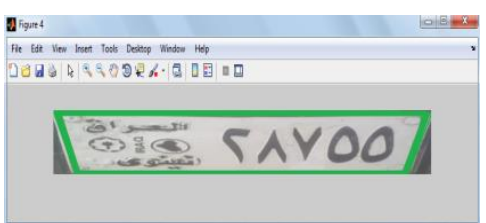

الثكل(6) لوحة تسجيل المركبة المقتطعة

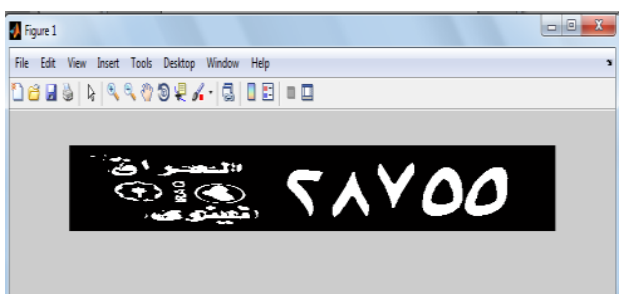

الثكل (8) الصورة بعد إزالة الإطار واخذ المتمم

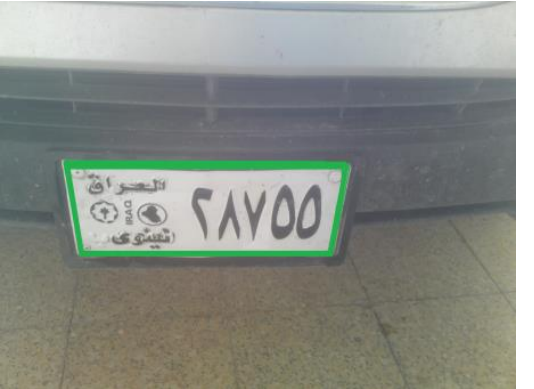

الثكل(5) صورة المركبة المراد تمييزها

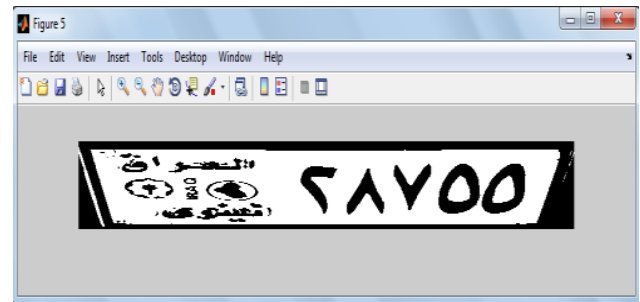

الثكل(7) الصورة بعد تحويلها إلى الصورة الثنائية

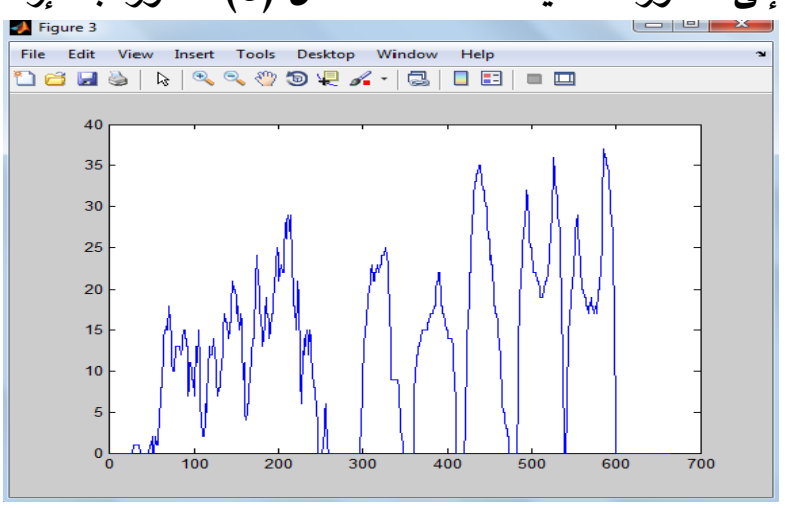

الثكل(9) المدرج التكراري لصورة لوحة التسجيل المقتطعة

من المدرج التكراري يتم فصل الجزء الأيمن الذي يمثل الأرقام عن الجزء الخاص باسم البلد والمدينة، انظر لهرجه

الثكل (10) الذي يوضح جزء الأرقام المقتطع، بينما الثكل (11) يوضح الصورة بعد الائ عملية التتحيف.

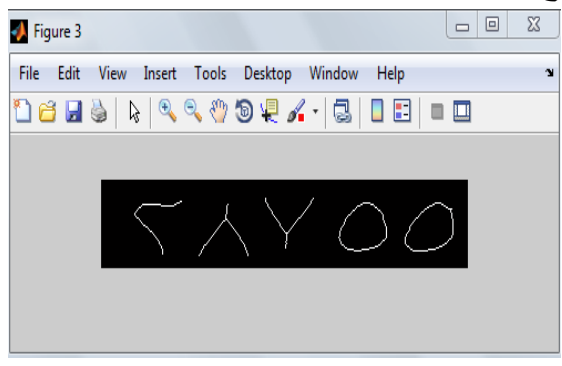

الشكل (11) الصورة بعد عملية التنحيف

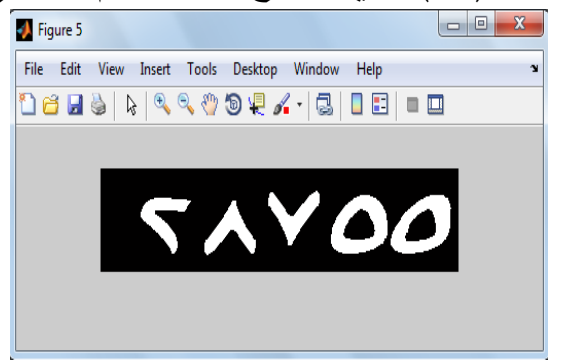

الثكل (10) مقطع صورة لوحة التسجيل الخاص بالأرقام 
أما الثكل(12) صور الأرقام بعد قطعها من صورة لوحة الأرقام، ونلاحظ في الثكل(13) الأقسام الستة عشر (4×4) لكل صورة رقم. والجدول (6) يبين الصيغة الثنائية لصورة كل رقم الذي سيتم مقارنتها مع قاعدة البيانات

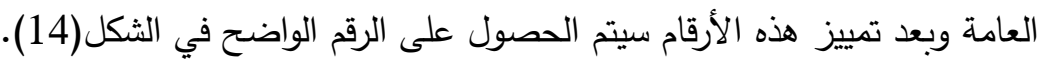

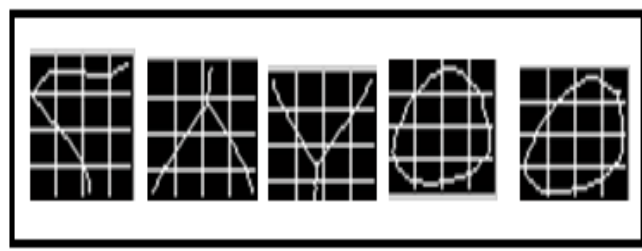

الثكل(13) تقسيم كل صورة إلى 16 جزء

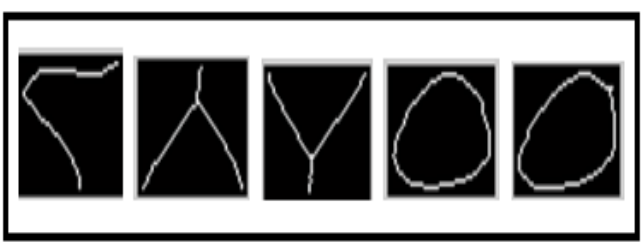

الثكل(12) صور الأرقام بعد قطعها

الجدول (6):الصيغة الثنائية لصورة كل رقم.

\begin{tabular}{|c|c|c|c|c|c|c|c|c|c|c|c|c|c|c|c|c|c|c|c|}
\hline 1 & 1 & 1 & 1 & 0 & 0 & 1 & 0 & 1 & 0 & 0 & 1 & 0 & 1 & 1 & 1 & 0 & 1 & 1 & 1 \\
\hline 0 & 1 & 1 & 0 & 0 & 1 & 1 & 0 & 1 & 1 & 1 & 1 & 1 & 0 & 0 & 1 & 1 & 1 & 0 & 1 \\
\hline 0 & 1 & 1 & 0 & 1 & 1 & 0 & 1 & 0 & 1 & 1 & 0 & 1 & 0 & 0 & 1 & 1 & 0 & 0 & 1 \\
\hline 0 & 0 & 1 & 0 & 1 & 0 & 0 & 1 & 0 & 1 & 0 & 0 & 1 & 1 & 1 & 1 & 1 & 1 & 1 & 1 \\
\hline
\end{tabular}

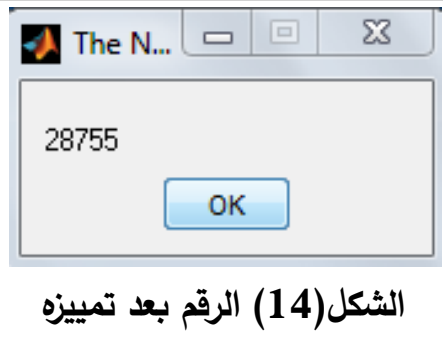

9

1- من الجدول(1) و الجدول(2) نلاحظ أن الرقم (0 ) ظهر بعدة أشكال وكذلك الرقم (1) والرقم (2) وهكذا حتى الرقم (9) وان سبب ظهور أشكال مختلفة من شكل الرقم هو اختلاف نوع وحجم الخط المستخدم بالكتابة. 2- إن طريقة مطابقة القوالب تعد من طرائق تمييز الأنماط التي تتأثر بتشوهات الصورة في عملية المطابقة، وقد تم الاستفادة من هذه الفكرة وإزالة تأثير التثوهات في الصورة من خلال تجزئة الصورة إلى 16 جزء ثم تحويل كل جزء إلى القيمة واحد أو صفر بغض النظر عن حجم الصورة، وبذلك فان كل قيمة واحد في المصفوفة الناتجة (التي سيتم مطابقتها مع القوالب المخزونة) قد نابت عن مجموعة قيم قد تكون هذه القيم مختلفة (أي قيمها 0 أو 1) نتيجة التثوهات إلا انه سيعوض عنها بالقيمة واحد. وللتقليل أكثر من تأثير التشوهات يمكن أن يكون مقارنة مجموع قيم كل جزء في الصورة مع قيمة اكبر من الصغر (فكلما كبرت هذه القيمة سيتم الاقتراب أكثر من التعميم أي التقليل من تأثير التشوهات) • 3- إن عملية التمديد التي سبقت عملية التتحيف أدت إلى تحسين شكل الرقم بعد عملية التتحيف إذ عالجت بعض التشوهات مثل الفراغات وأعادت بعض الحواف المتآكلة للرقم مما أدى إلى تتبيت المعالم العامة لشكل 
4- في عملية تحليل صفات الثكل لكل رقم، لوحظ انه يوجد صعوبة في بناء نموذج يعتبر كقاعدة قرار رصينة لتمييز كل رقم جديد يتم إدخاله وذلك لوجود بعض التثوهات المتمثلة بوجود خلايا(نقاط) ضوئية في صورة الرقم لا تتطبق بشكل كامل مع صورة في قاعدة البيانات. لذا تم تجزئة الصورة إلى ستة عشر جزءاً بحجم

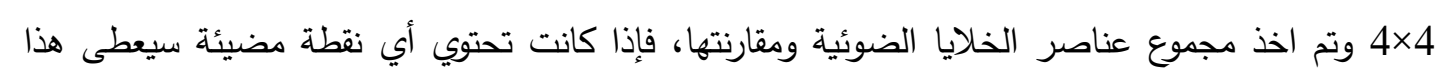
الجزء القيمة '1' وإلا سيعطى القيمة '0' وبذلك تم التخلص من مشكلة تشوه الثكل.

5- لوحظ من تطبيق الخوارزمية المقترحة على لوحة تسجيل المركبات انه من المحتمل أن تكون عملية التمييز غير دقيقة وذلك لاحتمال وجود بعض التشوهات في الرقم الناتجة من الصورة الملتقة بالكاميرا بسبب وجود

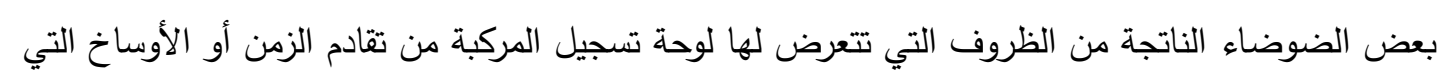
يمكن أن تلتصق على لوحة تسجيل المركبة وكذلك يمكن أن يكون وميض الضوء الناتج من الكاميرا أن يشوه الصورة المتتطة والذي اثر على شكل الأرقام في الصورة. لذا يجب أن تكون الأرقام واضحة ونظيفة. 6- في شكل الخط الأندلسي يكون شكل رقم 2و 3 متثابهان في التمثيل الثنائي، انظر الجدول(4) الصف الثاني مما يؤدي إلى عدم دقة التمييز • ولا يعد ذلك مؤثراً في الخوارزمية المقترحة إذ يمكن التخلص من هذه المشكلة بتقسيم صورة الرقم إلى 25 جزء (5×5). فكلما زاد عدد الأجزاء كانت نتيجة التمييز أدق. 7- تم تطبيق الخوارزمية المقترحة على 40 صورة للأرقام العربية من ضمنها 10 صور للوحات أرقام مركبات مختلفة تم التقاطها بكاميرات مختلفة وبدقات مختلفة. وباقي الصور هي لعشرة أنواع مختلفة لخطوط الأرقام من برنامج معالجة النصوص(Microsoft Word) وكل نوع بثلاثة أحجام من الخط هي (28و (36و728) منها الغامقة والاعتيادية. وكانت نتيجة التمييز 98\% حسب المعادلة (1)، إذ كانت عدد الصور المميزة 39 والصورة التي لم يتم تمييز بعض الأرقام فيها كانت لنوع الخط الأندلسي المائل. نسبة التمييز العدد الكلي للصور المطبقة المبلة 10.

بما انه تم الاعتماد في عملية التمييز على إدخال المعطيات المتمثلة بالصفات المميزة لصورة كل رقم وتم تصنيف البيانات حسب تثابهها مع المعطيات أو النماذج في قاعدة البيانات العامة لذا تعد هذه الطريقة هي إحدى لإلى طرائق التمييز المرشد(supervised recognition). إن الهدف الأساسي من اختيار الخصائص هو إيجاد دلالات يمكن من خلالها وصف الصورة التي يمكن أن تكون

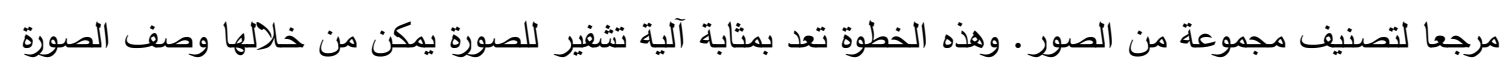
بعدد محدد من الدلالات غير المرتبطة. يمكن الاستفادة من الخوارزمية المقترحة في الأنظمة الأمنية الخاصة بحماية المنشات للسماح مثلا بإذخال المركبات المخولة التي تم تمييز رقمها إلى المناطق المحمية. 


\section{(المصادر}

[1] Cardoso, Angelo and Wichert, Andreas " Handwritten digit recognition using biologically inspired features", Neurocomputing, Vol. 99, pp 575-580, 1 January (2013).

صلاح الدين، جمال و طه، طارق حازم و علي، اسيل وليد ، "تمييز حروف المسند باستخدام

الخوارزميات الجينية"، المجلة العراقية للعلوم الإحصائية، المجلد 11، العدد 20، (2011). عبدالله، هدية صالح، "تمييز الصور باستخدام المطابقة القالبية المعتمدة على معامل الارتباط"، مجلة

$$
\text { التربية والعلم ، المجلد 23، العدد2، (2010). }
$$

طليع، غادة ذنون و بدران، عامرة استقلال و خضر، ميسون، "تمييز الحروف العربية باستخلاص خواصها اعتمادا على شبكتي الانتثار العكسي والمدرك والمقارنة بين اداء الثبكتين" ، المجلة

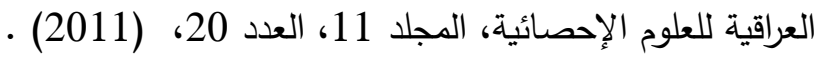

مصطفى، فدوى صبحي والنعمة، رائد رافع ، "تمييز الوجوه بالاعتماد على خصائص العزوم الثابتة"، مجلة تكريت للعلوم ،جامعة تكريت،، المجلد 2، العدد 14، ص 253-259، (2009).

لمى اكرم عبدالله، "تمييز اللهب في الصور الرقمية الملونة"، رسالة ماجستير ، جامعة الموصل، كلية

$$
\text { علوم الحاسوب والرياضيات، قسم علوم الحاسوب، (2002). }
$$

البدراني، هدية صالح عبد الله، "تمييز الحروف العربية المكتوبة بنظام word 7 "، بحث ماجستير،

كلية علوم الحاسبات والرياضيات، جامعة الموصل، (2001) ـ

[8] Ibrahim, Laheeb M., "Arabic Printed_Hand Written Character Recognition Using ANN", Ph.D thesis , Mosul University Computer Sciences And Mathematics, (2002).

ذنون، كرم حاتم،"تمييز الحرف العربي المطبوع باستخدام البعد الكسري" ، بحث ماجستير ، كلية علوم

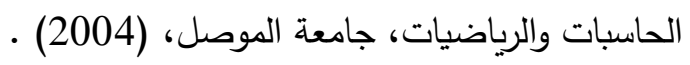

[10] Hamdey Hanan Zeki, "License Plate Recognition For Security Places", Journal Of Education And Science, Vol.22, No.3, Pp 92-108,(2009).

الاهدل، طارق عبدالله احمد، "تمييز انماط الارقام العربية والمستخدمة على نموذج الامر -المامور

باستخدام مفاهيم برمجيات الربط بين الثبكات" ، رسالة ماجستير ، جامعة بترا، ماليزيا، (2009). الزبيدي، لهيب محمد ابراهيم و الدليمي، حنان حامد على، "استخدام الثبكة العصبية الاصطناعية

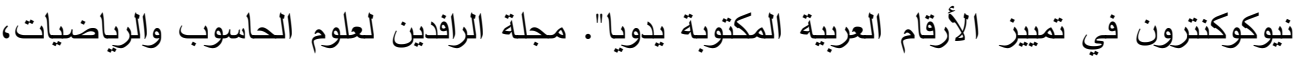

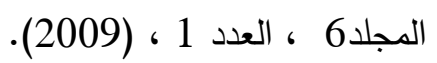

[13] Gohil, Naikur Bharatkumar Gohil, "Car License Plate Detection" M.Sc., California State University, Sacramento, (2010).

الطائي، خالد ضاري عباس والمرحوم عتاب، محمد رمضان، "استخدام الدالة المميزة التربيعية في

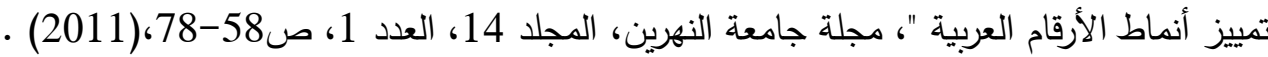
العبيدي، سندس خليل و العطيوي، رهام جاسم، "تمييز العلامات المرورية باستخدام الترميز التسلسلي"، مجلة الرافدين لعلوم الحاسوب والرياضيات، المجلد 9، العدد 2، (2012). 